\title{
Estimativa do volume de Acacia mangium utilizando técnicas de redes neurais artificiais e máquinas vetor de suporte
}

\author{
Márcio Assis Cordeiro ${ }^{1 *}$, Nayara Natacha de Jesus Pereira², Daniel Henrique Breda Binoti ${ }^{3}$, Mayra Luiza Marques da Silva Binoti ${ }^{4}$, \\ Hélio Garcia Leite ${ }^{3}$ \\ ${ }^{1}$ Amcel Floresta e Celulose, Rua Cláudio Lúcio Monteiro, 818, Hospitalidade, Novo Horizonte, CEP 68925-000, Santana, AP, Brasil \\ ${ }^{2}$ Universidade Federal de Minas Gerais, Instituto de Ciências Agrarias, Avenida Universitária, 1000, CEP 39000-000, Montes Claros, MG, Brasil \\ ${ }^{3}$ Universidade Federal de Viçosa, Avenida Peter Henry Rolfs, s/n, Campus Universitário, CEP 36570-900, Viçosa, MG, Brasil \\ ${ }^{4}$ Universidade Federal do Espírito Santo, Av. Governador Lindemberg, 316, Centro, CEP 29550000, Jerônimo Monteiro, ES, Brasil
}

"Autor correspondente:
marcio_irati@hotmail.com

Termos para indexação:

Smalian

Modelagem

Estimativa volumétrica

Index terms:

Smalian

Modeling

Volumetric estimates

Histórico do artigo:

Recebido em 27 set 2013

Aprovado em 28 maio 2015

Publicado em 30 set 2015

doi: $10.4336 / 2015 . p f b .35 .83 .596$
Resumo - Com o presente trabalho objetivou-se mostrar os resultados das estimativas volumétricas de Acacia mangium, obtidas pelo modelo de Schumacher e Hall, comparando-os com as metodologias de aplicação de redes neurais artificiais e máquinas vetor suporte. Para que fosse possível essa análise comparativa, foram utilizados dados de cubagens de 31 árvores de povoamentos de Acacia mangium, localizados no norte do estado do Amapá. Os dados apresentavam idade variando de 14 a 17 anos. As árvores-amostra foram cubadas em seções relativas, realizando medições de diâmetros e espessuras das cascas ao longo do fuste em 14 seções, baseando-se nos seguintes percentuais das alturas totais: $0,05 \%, 1 \%, 5 \%, 10 \%, 15 \%, 20 \%, 30 \%, 40 \%, 50 \%$, $60 \%, 70 \%, 80 \%, 90 \%$ e $95 \%$, sendo o volume total com casca obtido pela aplicação da fórmula de Smalian. De modo geral, as metodologias que diferem da tradicional apresentaram resultados estatisticamente superiores.

\section{Estimate of Acacia mangium volume using techniques of artificial neural networks and support vector machines}

\begin{abstract}
The present study aimed to show the results of Acacia mangium volumetric estimates obtained through the Schumacher and Hall model compared to the methods of artificial neural networks and support vector machines. To enable this comparative analysis, we used data from 31 trees of Acacia mangium aged 14-17, from a stand located in the northern region of the state of Amapá. Diameter and bark thickness of the trees were measured into relative heights along the stem into 14 sections $(0.05 \%, 1 \%, 5 \%, 10 \%$, $15 \%, 20 \%, 30 \%, 40 \%, 50 \%, 60 \%, 70 \%, 80 \%, 90 \%$, and $95 \%$ ), with measurement. Total volume with bark was obtained by applying the Smalian formula. In general, the methods that differ from traditional methods showed statistically superior results.
\end{abstract}




\section{Introdução}

Acacia mangium é uma espécie arbórea de rápido crescimento, rústica e tolerante a uma ampla gama de tipos de solos e ambientes (Newaz \& Mustafa, 2004). No Brasil essa espécie é popularmente conhecida como acácia, sendo sua madeira usualmente empregada na produção de celulose, papel, chapa de partículas e aglomerados. Apresenta também grande potencial para produção de madeira serrada, ligas, móveis, lenha e carvão, em função do seu poder calorífico (entre 4800-4900 Kcal kg-1) (Krisnawati et al., 2011), além da serragem que fornece substrato de boa qualidade para cogumelos comestíveis (Lim et al., 2003).

A espécie destaca-se também pelos seus usos não madeireiros, que incluem a produção de cola e mel. As árvores são úteis para a sombra, fins ornamentais, e quebra-ventos, sendo também cultivadas em sistemas agroflorestais e para controle de erosão (National Research Council, 1983; Müller et al., 2009).

A quantificação do estoque e do crescimento é fundamental para o manejo florestal, por fornecer informações quantitativas e qualitativas, auxiliando a avaliação técnica e econômica dos plantios florestais. Esta quantificação baseia-se na estimação do volume de árvores amostradas através da aplicação de equações volumétricas, razão volumétrica, dentre outras e técnicas de inteligência artificial (Campos \& Leite, 2009; Silva et al., 2009; Leite et al., 2011; Binoti, 2012). Devido à importância e ao valor econômico que a acácia apresenta, diversos modelos estatísticos foram testados para estimação do volume de plantios dessa espécie, (Sumarna \& Bustomi, 1986; Bustomi, 1988; Krisnawati et al., 1997; Müller et al., 2009).

O desenvolvimento e aplicação de diferentes abordagens para a quantificação do volume de árvores é impulsionado pelo fato que pequenos ganhos em exatidão em nível de árvore representam ganhos significativos quando contabilizados os valores totais do povoamento. Diversas ferramentas e metodologias têm sido introduzidas na área florestal visando à redução de custos e aumento de exatidão das estimativas (Campos \& Leite, 2009; Binoti, 2012). Dentre essas ferramentas de inteligência computacional (IC), as técnicas de redes neurais artificiais (RNA) e máquinas de vetores de suporte (MVS), têm despertado interesse na área florestal (Binoti, 2012).

Os modelos matemáticos baseados em técnicas de inteligência artificial, como as RNA e MVS, apresentam como principal vantagem independência de conhecimento e suposições sobre as relações entre as variáveis utilizadas no ajuste. Outra vantagem referese à utilização de variáveis categóricas e numéricas em um mesmo modelo. Essas vantagens, em relação aos modelos de regressão, têm motivado a aplicação de IC em diferentes problemas na área florestal (Guan \& Gertner, 1991; Schmoldt et al., 1997; Blackard \& Dean, 1999; Zhang \& Zhang, 2000; Leduc et al., 2001; Liu et al., 2003; Corne et al., 2004; Diamantopoulou, 2005; Avramidis et al., 2006; Silva, 2008; Görgens et al., 2009; Leite et al., 2011; Binoti, 2012).

Redes neurais artificiais são sistemas de processamento de informação que possuem características que se assemelham com as redes neurais biológicas. As RNA foram desenvolvidas como generalizações de modelos matemáticos da cognição humana e baseiam-se em: 1) Informações processadas em uma grande quantidade de simples elementos, chamados de neurônios; 2) Os sinais são passados entre neurônios através de conexões; 3) Cada conexão possui um peso associado, o qual, em uma RNA típica, é multiplicado pelo sinal transmitido; 4) Cada neurônio possui uma função de ativação (linear ou não linear) que determina a saída do neurônio em função do somatório dos pesos e sinais recebidos.

Uma RNA é caracterizada em função da sua: a) arquitetura, que se refere ao padrão de conexões entre os neurônios; b) treinamento, ou aprendizagem, que determinam os pesos associados às conexões; e c) a função de ativação (Fausett, 1993).

Máquinas de vetores de suporte (MVS) tem se mostrado como uma alternativa interessante para a modelagem matemática de sistemas complexos. São técnicas simples em sua base conceitual e com capacidade de resolução de problemas reais extremamente complexos. Em comparação com as RNA, tem a vantagem de conduzir a uma solução exata, ou seja, a um ótimo global. No entanto, encontrar um modelo final de MVS pode apresentar complexidade computacional, porque exige a solução de um modelo de programação quadrática e a solução de um conjunto de equações não lineares.

Inicialmente, as técnicas de MVS foram utilizadas como uma metodologia de classificação. Posteriormente foram estendidas para as tarefas de regressão através de duas abordagens: support vector regression (SVR) e least-squaressupport vector machines (LS-SVMs). Exemplos de aplicações dessas técnicas podem ser 
encontrados em Drucker et al., (1997); Cherkassky \& Mulier (1998); Schölkopf et al., (1998); Vapnik (1998), (1999); Mattera \& Haykin (1999); Schölkopf \& Smola (2002); dentre outros).

Neste estudo objetivou-se utilizar as técnicas de MVS e RNA para a estimação do volume de árvores de Acacia mangium, bem como comparar os resultados obtidos com modelos volumétricos comumente utilizados na área florestal.

\section{Material e métodos}

\section{Dados}

Foram utilizados dados de cubagem de 31 árvores de povoamentos de Acacia mangium, localizados no norte do estado do Amapá. As árvores apresentavam idade variando de 14 a 17 anos.

As árvores-amostra foram cubadas em seções relativas, com medições de diâmetro e espessura da casca ao longo do fuste em 14 seções, baseando-se nos seguintes percentuais das alturas totais: $0,05 \%, 1 \%, 5 \%$, $10 \%, 15 \%, 20 \%, 30 \%, 40 \%, 50 \%, 60 \%, 70 \%, 80 \%, 90 \%$ e $95 \%$. O volume total com casca $(V)$ foi obtido pelo método de Smalian, utilizando a equação 1 (Machado \& Figueiredo Filho, 2003):

$$
\mathrm{V}_{\mathrm{i}}=\frac{\left(g_{1}+g_{2}\right)}{2} * \mathrm{~L}
$$

Onde:

$\mathrm{V}=$ volume da seção $\mathrm{i}$ da tora $\mathrm{em}^{3} ; \mathrm{g}_{1}=$ área transversal na base da seção i da tora $\left(\mathrm{m}^{2}\right) ; \mathrm{g}_{2}=$ área transversal no topo da seção i da tora $\left(\mathrm{m}^{2}\right) ; \mathrm{L}=$ comprimento da seção da tora $(\mathrm{m}) ; \mathrm{i}=$ número de seções da tora $(i=1,2,3, \ldots ., n)$.

Desta forma, o volume total da árvore pode ser determinado pelo somatório do volume das diferentes seções mais o volume da ponta e do toco.

A base de dados foi construída de acordo com os parâmetros representados na tabela 1, onde são apresentadas as variáveis consideradas. A dispersão do volume com e sem casca em função do diâmetro a 1,3 m do solo (dap) está apresentado na Figura 1.

Tabela 1. Dados de diâmetro, altura e volume utilizados.

\begin{tabular}{lcccc}
\hline \multicolumn{1}{c}{ Parâmetro } & Mínimo & Médio & Máximo & $\begin{array}{c}\text { Desvio } \\
\text { padrão }\end{array}$ \\
\hline Dap $(\mathrm{cm})$ & 8,20 & 15,21 & 23,00 & 4,35 \\
Altura $(\mathrm{m})$ & 11,00 & 17,85 & 25,00 & 3,35 \\
Volume total com casca $\left(\mathrm{m}^{3}\right)$ & 0,0309 & 0,2083 & 0,4884 & 0,1305 \\
Volume total sem casca $\left(\mathrm{m}^{3}\right)$ & 0,0254 & 0,1809 & 0,4330 & 0,1157 \\
\hline
\end{tabular}

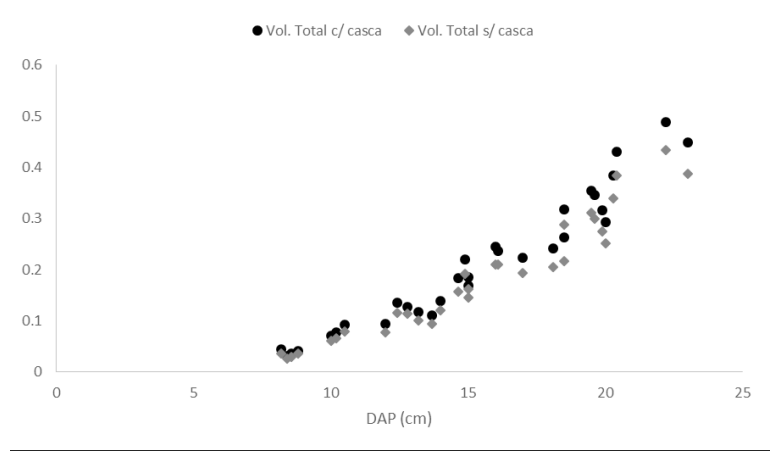

Figura 1. Relação entre volume com e sem casca em função do dap para os dados utilizados no estudo.

\section{Modelo volumétrico}

As estimativas volumétricas foram obtidas pela aplicação do modelo de Schumacher e Hall, convencionalmente utilizado na área florestal (equação 2).

$$
\text { Volume }=\beta_{0} * D A P^{\beta_{1}} * H t^{\beta_{2}} * \varepsilon
$$

Em que,

$\mathrm{V}=$ volume $\left(\mathrm{m}^{3}\right) ; \mathrm{DAP}=$ diâmetro a $1,3 \mathrm{~m}$ de altura $(\mathrm{cm}) ; \mathrm{H}=$ altura total da árvore $(\mathrm{m}) ; \beta_{0}=$ parâmetros; e $\varepsilon=$ erro aleatório.

\section{Máquinas de vetores de suporte e redes neurais artificiais}

A construção de máquinas de vetores se baseou no processo de aprendizado estatístico descrito em detalhes por Haykin (2001) e por Steinwart \& Christmann (2008). Utilizou-se como função de erro a função tipo II, também conhecida como nu-SVM, sendo a função kernel do tipo RBF (função de base radial). As funções Kernel oferecem uma solução alternativa ao projetar os dados num espaço com características de alta dimensão, ou seja, para aumentar o poder computacional das máquinas de aprendizagem lineares, possibilitando representar fenômenos não lineares (Cristianini \& Shawe-Taylor, 2000).

As RNA treinadas foram do tipo perceptrons de múltiplas camadas, comumente conhecidas como MLP (Multilayer Perceptron). Como função de ativação utilizou-se a função sigmoidal. Utilizou-se o algoritmo de treinamento Resilient Propagation. A parada do treinamento foi realizada pela técnica $k$-foldcrossvalidation (Fausett, 1993). 
A construção das MVS e RNA foi feita utilizandose o aplicativo NeuroForest - 3.5 (Binoti, 2015). No presente trabalho utilizou-se como variáveis independentes contínuas o dap $(\mathrm{cm})$ e a altura-Ht $(\mathrm{m})$, e como variáveis dependentes o volume total com e sem casca. As variáveis foram normalizadas pela técnica de normalização equilateral (Heaton, 2011).

\section{Avaliação dos ajustes}

Os volumes estimados pelo modelo de Schumacher e Hall, MVS e RNA foram comparados com os volumes observados. As estimativas foram analisadas empregando-se estatísticas e análise gráfica de resíduos. A análise gráfica consistiu na inspeção estatística da dispersão dos erros (resíduos) percentuais em relação aos valores observados, relação entre valores observados e estimados, bem como na análise do histograma de resíduos. A estatística empregada foi a correlação entre os volumes estimados e observados, considerando:

$r_{Y \hat{Y}}=\frac{\operatorname{cov}(Y, \hat{Y})}{\sqrt{s^{2}(Y) s^{2}(\hat{Y})}}$

em que $\mathrm{s}^{2}$ é a variância e cov é a covariância.

\section{Resultados}

Todos os modelos testados apresentaram resultados sem tendenciosidade e livre de bias (erro de tendência). Os coeficientes de correlação obtidos por cada técnica e sortimento são apresentados na Tabela 2.

Tabela 2. Coeficientes de correlação (r) para as metodologias testadas.

\begin{tabular}{lcc}
\hline \multirow{1}{*}{ Metodologia } & \multicolumn{2}{c}{$\boldsymbol{r}_{\boldsymbol{Y \hat { Y }}}$} \\
\cline { 2 - 3 } & $\begin{array}{c}\text { Volume com } \\
\text { casca }\end{array}$ & $\begin{array}{c}\text { Volume sem } \\
\text { casca }\end{array}$ \\
\hline Schumacher e Hall & 0.9661 & 0.9533 \\
Rede neural artificial & 0.9940 & 0.9888 \\
Máquina vetor de suporte & 0.9857 & 0.9832 \\
\hline
\end{tabular}

A análise gráfica dos ajustes do modelo de Schumacher e Hall, MVS e RNA está apresentada na Tabela 3. Observa-se pelo histograma de resíduos uma maior dispersão para os modelos de Schumacher e Hall.

Nas Figuras 2 e 3 são apresentados os volumes observados e estimados pelo modelo de Schumacher e Hall, e utilizando-se as técnicas de RNA e MVS em função do dap.

Tabela 3. Relação entre valores observados e estimados, dispersão de resíduos em função do volume observado e histograma de erros para volumes com e sem casca.

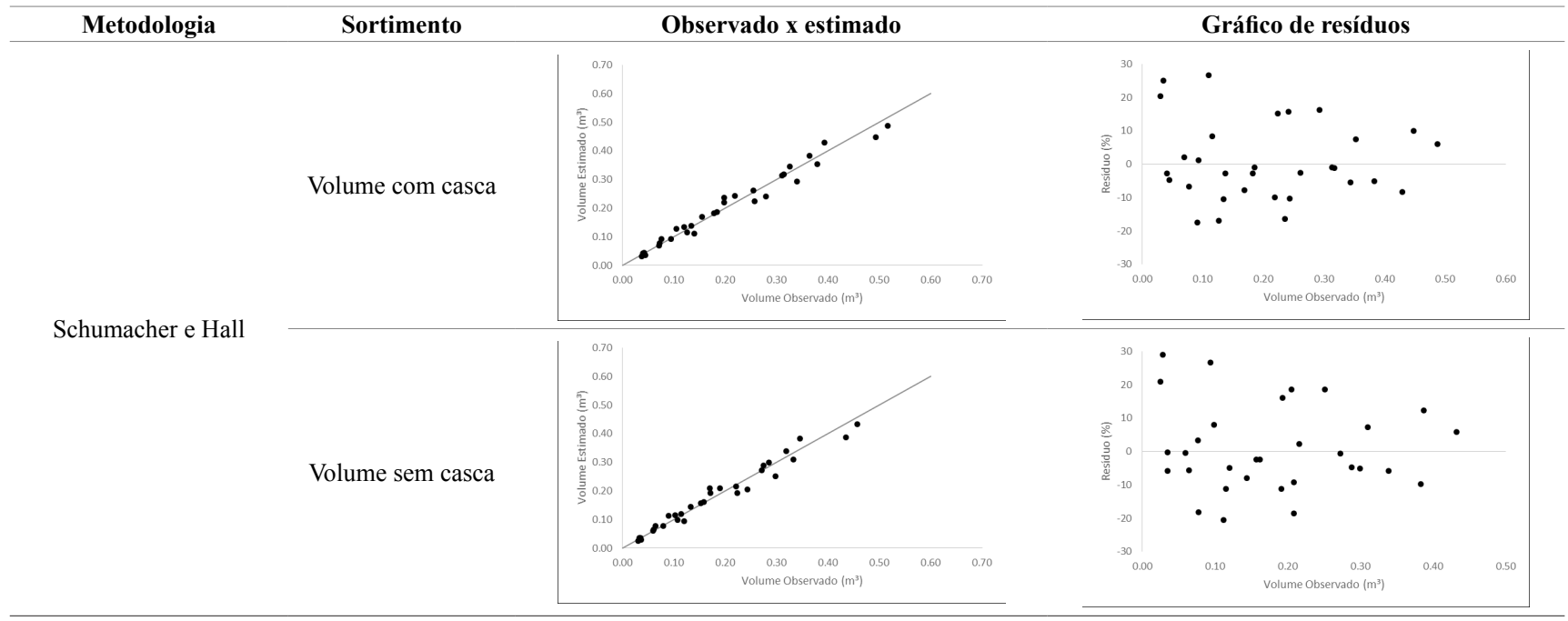


Tabela 3. continuação.

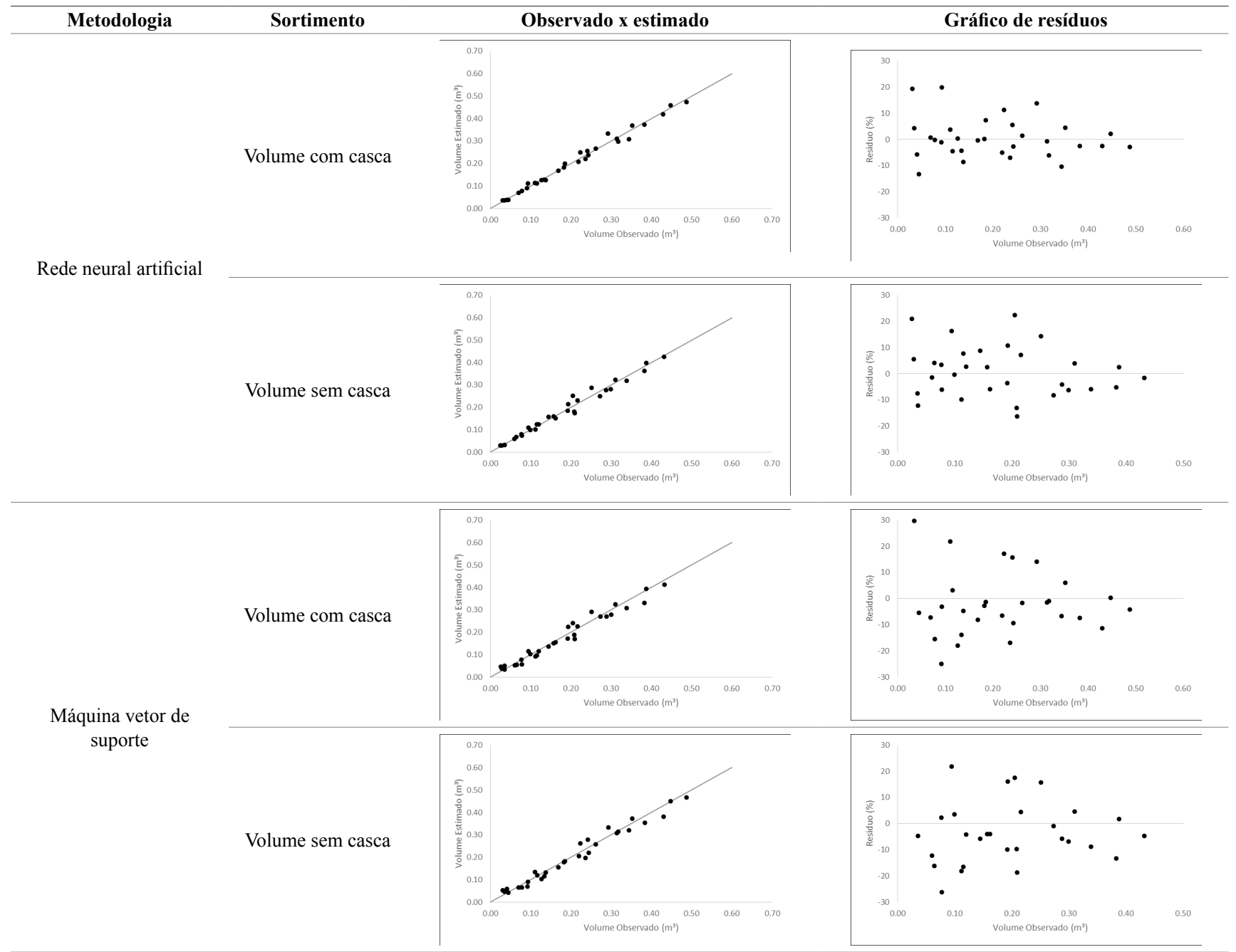

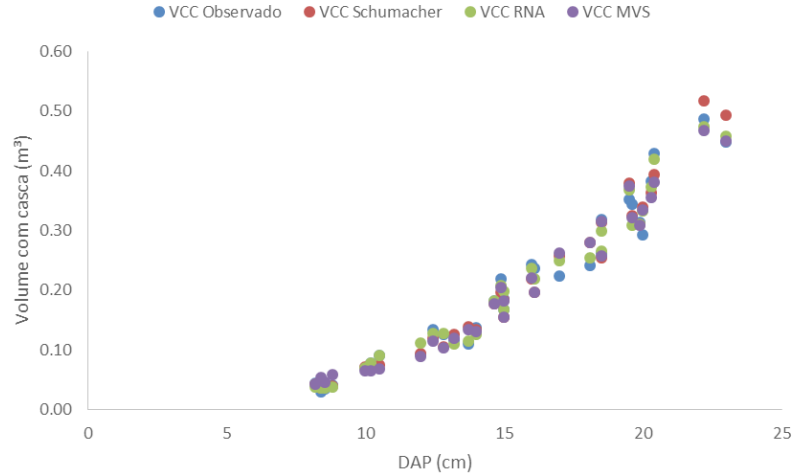

Figura 2. Comparativo entre volume observado com casca e valores estimados pelo modelo de Schumacher e Hall, RNA e MVS.

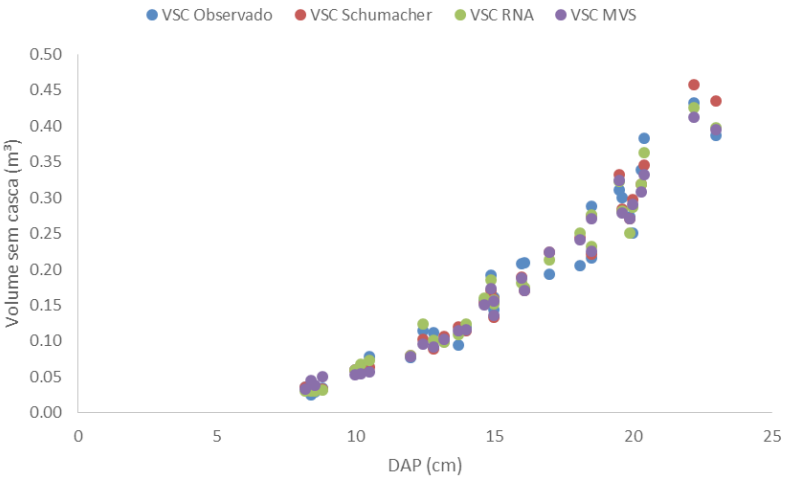

Figura 3. Comparativo entre volume observado sem casca e valores estimados pelo modelo de Schumacher e Hall, RNA e MVS. 


\section{Discussão}

A análise gráfica e estatística utilizada mostrou que todos os modelos apresentaram resultados sem tendenciosidade e livre de bias. As técnicas de inteligência artificial apresentaram coeficientes de correlação superior ao modelo de Schumacher e Hall, contudo tal diferença não se apresenta significativa para os dados em questão. Deve-se considerar o fato de utilização dessas técnicas para problemas maiores e mais complexos, onde essa diferença pode ser significativa.

Considerando as técnicas de inteligência artificial, uma das principais diferenças entre o RNA e MVS refere-se às gerações de soluções a partir de uma mesma base de dados. Enquanto que para a RNA pode haver muitas soluções, o SVM converge para uma única solução ótima (Haykin, 2001).

Com base nas estatísticas apresentadas e análise gráfica dos resíduos, as RNA apresentaram um melhor desempenho em relação às MSV e ao modelo de Schumacher e Hall. Nesse trabalho foram utilizadas parametrizações das RNA obtidas por Binoti (2012) para estimação do volume de clones de eucalipto, contudo parametrizações das MVS para a estimação do volume de árvores não são disponíveis na literatura, e em função disso utilizou-se uma configuração comumente utilizada para aplicações gerais (Suykens et al., 2002).

Em função da pouca quantidade de dados disponíveis para ajuste dos modelos volumétricos, a parametrização e avaliação de outras configurações das MVS não foram possíveis. Em estudos comparativos, as técnicas de MVS apresentam-se superiores quando comparadas à obtenção tradicional das RNAs (Haykin, 2001; Valença, 2007).

Outro aspecto importante é que o uso das MVS tem por objetivo principal obter uma ferramenta com alta capacidade de generalização, uma vez que, durante o treinamento destas, se busca não apenas minimizar o erro de treinamento, mas também a complexidade desta (Valença, 2007). Diversas aplicações utilizando as MVS para aproximação de funções são encontradas na literatura (Drucker et al., 1997; Cherkassky \& Mulier, 1998;; Schölkopf et al., 1998, 1999; Smola \& Schölkopf, 1998; Vapnik, 1998, 1999; Mattera \& Haykin, 1999; Schölkopf \& Smola, 2002).

Este trabalho apresenta-se como introdutório às técnicas MVS e RNA para a obtenção de estimativas volumétricas em plantios de $A$. mangium. Sugere-se a condução de outros trabalhos de parametrização destas técnicas bem como sua aplicação em outros problemas da área florestal.

\section{Conclusões}

O uso da técnica de RNA para estimativa de volume com e sem casca obteve maior precisão dos resultados quando comparado ao modelo usual de Schumacher e Hall.

Quanto à adequação ao problema, as técnicas de MVS e RNA apresentaram-se satisfatórias, possibilitando a utilização para previsão volumétrica da produção de florestas plantadas de Acacia Mangium.

\section{Referencias}

AVRAMIDIS, S.; ILIADIS, L.; MANSFIELD, S. D. Wood dielectric loss factor prediction with artiûcial neural networks. Wood Science Technology, v. 40, n. 7, p. 563-574, 2006.

BINOTI, D. H. B. Neuroforest. Disponível em $<$ http://neuroforest. ucoz.com>. Acesso em: 5 jan. 2015.

BINOTI, M. L. M. da S. Redes neurais artificiais em mensuração e manejo florestal. 2012. Tese (Doutorado em Ciência Florestal) Universidade Federal de Viçosa, Viçosa, MG.

BINOTI, M. L. M. da S. Redes neurais artificiais para prognose da produção de povoamentos não desbastados de eucalipto. 2010. 54 f. Dissertação (Mestrado em Ciência Florestal) - Universidade Federal de Viçosa, Viçosa, MG.

BLACKARD, J.; DEAN, D. Comparative accuracies of artiûcial neural networks and discriminant analysis in predicting forest cover types from cartographic variables. Computers and Electronics in Agriculture, New York, v. 24, n. 1, p. 131-151, 1999.

BUSTOMI, S. Tabel isi pohon lokal Acacia mangium Willd. untuk daerah Balikpapan Kalimantan Timur. Buletin Penelitian Hutan, v. 495, p. 31-38, 1988.

CAMPOS, J. C. C.; LEITE, H. G. Mensuração florestal: perguntas e respostas. 3 ed. Viçosa, MG: Ed daUFV, 2009.

CHERKASSKY, V.; MULIER, F. Learning from data: Concepts, theory, and methods. New York: Wiley, 1998.

CORNE, S. A.; CARVER, S. J.; KUNIN, W. E.; LENNON, J. J.; VAN HEES, W. W. S. Predicting forest attributes in southeast Alaska using artiûcial neural networks. Forest Science, v. 50, n. 2, p. 259 276, Apr. 2004.

CRISTIANINI, N.; SHAWE-TAYLOR, J. An introduction to support vector machines: and other kernel-based learning methods. New York: Cambridge University Press, 2000.

DIAMANTOPOULOU, M. J. Artificial neural networks as an alternative tool in pine bark volume estimation. Computers and Electronics in Agriculture, v. 10, n. 3, p. 235-244, 2005. DOI: 10.1016/j.compag.2005.04.002 
DRUCKER, H.; BURGES, C.; KAUFMAN, L.; SMOLA, A.; VAPNIK, V. Support vector regression machines. In: MOSER, M.; JORDAN, J.; PETSCHE, T. (Ed.). Neural information processing systems. Cambridge, MA: MIT Press, 1997. v. 9. P. 155-161.

FAUSETT, L. V. Fundamentals of neural networks: architectures, algorithms, and applications. Delhi: Prentice Hall, 1993.

GÖRGENS, E. B.; LEITE, H. G.; SANTOS, H. Do N.; GLERIANI, J. M. Estimação do volume de árvores utilizando redes neurais artificiais. Revista Árvore, Viçosa, MG, v. 33, n. 6, p. 1141-1147, 2009. DOI: $10.1590 / \mathrm{S} 0100-67622009000600016$

GUAN, B. T.; GERTNER, G. Modeling red pine tree survival with an artiûcial neural network. Forest Science, v. 37, n. 5, p.14291440, 1991.

HAYKIN, S. Redes neurais: princípios e prática. Porto Alegre: Bookman, 2001. 900 p.

HEATON, J. Programming neural networks with Encog3 in Java. 2nd ed. St Louis: Heaton Research, 2011. 240 p.

KRISNAWATI, H.; KALliO, M.; KANNINEN, M. Acacia mangium Willd.: ecology, silviculture and productivity. Jakarta: CIFOR, 2011.

KRISNAWATI, H.; WAHJONO, D.; IRIANTONO, D. Tabel isi pohon dan taper batang Acacia mangium Willd. di Kebun Benih Parungpanjang, Bogor, Jawa Barat. Buletin Teknologi Perbenihan, v. 4, p. 12-27, 1997.

LEITE, H. G.; SILVA, M. L. S; BINOTI, D. H. B.; FARDIN, L.; TAKIZAWA, F. H. Estimation of inside-bark diameter and heartwood diameter for Tectonagrandis Linn. trees using artificial neural networks. European Journal Forest Research, v. 130, n. 2, p. 263-269, 2011.

LIM, S. C.; GAN, K. S.; CHOO, K. T. The characteristics, properties and uses of plantation timbers - rubberwood and Acacia mangium. Timber Technology Bulletin, v. 26, p. 1-11, 2003.

LIU, C. et al. Comparison of neural network and statistical methods in classiûcation of ecological habitats using FIA data. Forest Science, v. 49, n. 4, p. 619631, 2003.

MACHADO, A. M.; FIGUEIREDO FILHO, A. Dendrometria. Curitiba, 2003. 309 p.

MATTERA, D.; HAYKIN, S. Support vector machines for dynamic reconstruction of a chaotic system. In: SCHÖLKOPF, B.; BURGES, C. J. C.; SMOLA, A. J. (Ed.). Advances in kernel methods: support vector machine. Cambridge, Mass: MIT Press, 1999.

MULLER, K.; SMOLA, A.; RATSCH, G.; SCHO” LKOPF, B.; KOHLMORGEN, J.; VAPNIK, V. Using support vector machines for time series prediction. In SCHÖLKOPF, B.; BURGES, C. J. C.; SMOLA, A. J. (Ed.). Advances in kernel methods: support vector machine. Cambridge, Mass: MIT Press, 2009.
NATIONAL RESEARCH COUNCIL. Risk Assessment in the Federal Government: managing the process. Washington, DC: National Academy Press 1983.

NEWAZ, S. M.; MUSTAFA, M. M Growth and yield prediction models for Acacia mangium grown in the plantations of the central region of Bangladesh. New Forests, v. 27, n. 1, p. 81-88, 2004.

SILVA, M. L. M. Estimação do diâmetro sem casca e diâmetro do cerne para árvores de Tectona grandis Linn. utilizando redes neurais artificiais. 2008. 47 f. Monografia (Graduação em Engenharia Florestal) - Universidade Federal de Viçosa, Viçosa, MG.

SCHÖLKOPF, B.; BARTLETT, P.; SMOLA, A.; WILLIAMSON, R. Support vector regression with automatic accuracy control. In: NIKLASSON, L.; BODEN, M.; ZIEMKE, T.(Ed.). Proceedings of ICANN'98: perspectives in neural computing. Berlin: Springer, 1998. p. 111-116.

SCHÖLKOPF, B.; BURGES, J.; SMOLA, A. Advances in kernel methods: support vector machine. Cambridge, Mass: MIT Press, 1999.

SCHÖLKOPF, B.; SMOLA, A. Learning with kernels: support vector machines, regularization, and beyond. Cambridge, Mass: MIT Press, 2002.

SCHMOLDT, D. L.; LI, P.; ABBOTT, A. L. Machine vision using artiûcial neural networks with local 3D neighborhoods. Computers and Electronics in Agriculture, v. 16, p. 255-271, 1997.

SILVA, M. L. M.; BINOTI, D. H. B.; GLERIANI, J. M.; LEITE, H. G. Ajuste do modelo de Schumacher e Hall e aplicação de redes neurais artificiais para estimar volume de árvores de eucalipto. Revista Árvore, Viçosa, MG, v. 33, n. 6, p. 1133-1139, 2009.

SMOLA, A.; SCHÖLKOPF, B. A tutorial on support vector regression. London: Royal Holloway College, University of London, 1998. (NeuroCOLT Technical Report NC-TR-98-030).

STEINWART, I.; CHRISTMANN, A. Support vector machines. Berlin: Springer, 2008.

SUMARNA, K.; BUSTOMI, S. Tabel isi pohon local Acacia mangium Willd. untuk daerah Subanjeriji, Sumatra Selatan. Buletin Penelitian Hutan, v. 487, p. 41-49, 1986.

SUYKENS, J. A.; GESTEL, T. V.; BRABANTER, J. D.; MOOR, B. D.; VANDEWALLE, J. . Least squares support vector machines. River Edge: World Scientific, 2002.

VALENÇA, M. J. S. Fundamentos das redes neurais: exemplos em Java. Recife: Livro Rápido, 2007. 382 p.

VAPNIK, V. Statistical learning theory. New York: Wiley. 1998.

VAPNIK, V. The nature of statistical learning theory. Berlin: Springer, 1999.

ZHANG, H. J.; ZHANG, A. I. Modeling treering growth responses to climatic variables using artiûcial neural networks. Forest Science, Bethesda, v. 46, n. 2, p. 229239, 2000. 
